\title{
The prevalence of naturally acquired multiple infections of Wuchereria bancrofti and human malarias in anophelines
}

\author{
T. R. BURKOT ${ }^{1,2}$, L. MOLINEAUX ${ }^{3}$, P. M. GRAVES ${ }^{1}$, R. PAR $U^{1}$, D. BATTISTUTTA ${ }^{2}$, \\ H. DAGORO ${ }^{1}$, A. BARNES ${ }^{4}$, R. A. WIRTZ ${ }^{5}$ and P. GARNER ${ }^{1}$ \\ ${ }^{1}$ Papua New Guinea Institute of Medical Research, P.O. Box 378, Madang, Papua New Guinea \\ ${ }^{2}$ Tropical Health Program, Queensland Institute of Medical Research, Bramston Terrace, Herston, Queensland 4006, \\ Australia \\ ${ }^{3}$ World Health Organization, $\mathrm{CH}-1211$ Geneva 27, Switzerland \\ ${ }^{4}$ Tropical Health Education Program, University of Queensland Medical School, Herston Road, Brisbane, Queensland \\ 4006, Australia \\ ${ }^{5}$ Walter Reed Army Institute of Research, Washington, D.C. 20307, USA
}

(Accepted 7 November 1989)

\begin{abstract}
SUMMARY
Malaria and filaria infection rates were determined for anopheline mosquitoes collected whilst biting and resting in village houses in Papua New Guinea. The number of anophelines infected with both parasites was greater than expected from the infection rates of each parasite and this difference was significant in resting collections. The excess of multiply infected mosquitoes is probably a result of a vector population composed of individuals with differing numbers of opportunities to become infected. Malaria-positive Anopheles punctulatus from resting catches had a significantly greater number of Stage 3 Wuchereria bancrofti larvae than malaria-negative mosquitoes. However, multiply infected mosquitoes appear to suffer greater mortality than non-infected or singly infected mosquitoes when the filarial worm reaches the third stage. Any potential increase in transmission resulting from multiple infections is thereby offset by a greater mortality rate in these mosquitoes.
\end{abstract}

Key words: multiple infections, Wuchereria bancrofti, Plasmodium falciparum, Plasmodium vivax, Anopheles punctulatus.

\section{INTRODUCTION}

The transmission of vector-borne diseases is dependent on the rate of transmission of the pathogen to the vector and from the vector back to a second host. The transmission rate to the vector depends on many factors including the degree of contact between pathogen, vector and host, the susceptibility of the vector to the pathogen, the effects of the pathogen on survivorship of host and vector and interactions between pathogens which may affect susceptibility of the vector to infection or the effect of parasite density on the vector.

Interactions between pathogens may be epidemiologically significant as naturally acquired concommitant infections are the norm for some parasites (e.g. murine malarias; Cox, 1978). Interactions between malaria and a second parasite have been studied more thoroughly in vertebrate hosts under laboratory conditions (reviewed by Cox, 1978 and Richie, 1988). Most of these studies have suggested that a second parasite has either a benign or a suppressive effect on malaria. Studies in chickens revealed no interactions between Plasmodium gallinaceum and Eimeria acervulina (Williams, 1985). Trypanosoma brucei-infected mice had longer prepatent periods when infected with Plasmodium chabaudi, but without affecting the resulting parasitaemias (Millott \& Cox, 1985). Microfilaraemic infections in owl monkeys, Aotus trivergatus griseimembra, generally result in more benign Plasmodium falciparum infections than in amicrofilaraemic monkeys (Schmidt \& Essinger, 1981). Important exceptions to this trend are seen in rodents infected with any of the following combinations of parasites: Plasmodium yoelii and Leishmania mexicana amazonensis (Coleman, Edman \& Semprevivo, 1988), or Plasmodium berghei and either Toxoplasma gondii, Trypanosoma lewisi or Trypanosoma musculi (reviewed by Cox, 1978). Such rodents suffer heavier parasitaemias and more severe clinical disease. In addition, mice with concomitant $P$. yoelii and L. $m$. amazonensis infections are more successfully fed upon by mosquitoes than are singly infected mice (Coleman et al. 1988).

A review of data from human malaria field studies (Richie, 1988) reveals a significant association between concurrent $P$. falciparum and $P$. malariae infections. However, the prevalence of the two parasite populations varies inversely, suggesting a suppressive effect in individuals either suffering greater exposure or susceptibility to malaria (Molineaux et al. 1980). Further evidence for a suppressive effect comes from the observation that a latent malaria infection will recrudesce immediately preceding or following a parasitaemic episode of a second malaria species.

Interactions of multiple pathogen species infec- 
tions in invertebrate vectors have been less frequently studied. Under laboratory conditions, Turrell et al. (1984) demonstrated that simultaneous ingestion of filarial worms with viruses could change a normally non-susceptible mosquito species into a vector by physical disruption of the gut barrier by microfilariae, thereby allowing the virus access to the haemocoel. As the mosquito midgut is a significant barrier to malaria infection (Ponnudurai, Billingsley \& Rudin, 1988), concurrent ingestion of microfilariae and malaria gametocytes could result in heavier infections.

In the laboratory, heavier malaria and filarial infections can significantly affect mosquito survivorship (Townson, 1971; Klein et al. 1986) and flight behaviour (Townson, 1970; Hockmeyer et al. 1975; Berry, Rowley \& Christensen, 1987; Rowland \& Boersma, 1988) with heavy filarial infections also affecting fecundity (Javadian \& MacDonald, 1974). Such laboratory models are often inappropriate for predicting factors affecting parasite transmission in endemic areas. Reasons for this include the use of vectors and parasites distributed allopatricly in nature or that the parasite densities used are many times greater than found in nature.

To elucidate whether these laboratory observations are epidemiologically significant for human malarias and filariasis, parasite prevalences and densities in naturally infected mosquitoes were studied. Infections in Anopheles punctulatus, a vector of human malarias and $W$. bancrofti in Papua New Guinea (Bryan, 1986; Burkot et al. 1989), were examined for interactions that may affect the transmission of either human malaria or filariasis.

\section{MATERIALS AND METHODS}

Mosquitoes were collected in Buksak village, Madang Province, Papua New Guinea. The village is hyperendemic for malaria, and Wuchereria bancroft $i$ microfilariae were found in 47 of 92 people screened by filtration of $2 \mathrm{ml}$ of venous blood samples collected between 22.00 and $01.00 \mathrm{~h}$.

Mosquito collections of a week's duration were conducted 14 times between January 1986 and March 1987. Mosquitoes were collected in landing and indoor resting catches, as described previously (Burkot et al. 1987, 1988a). Sentinel mosquito collectors ingested Maloprim and diethylcarbamazine weekly as prophylaxis against malaria and filariasis, respectively. Venous blood samples from mosquito collectors were screened for the presence of microfilariae before the study began, at the conclusion and 1 year after the study ended. All collectors were malaria and microfilariae negative. Collectors worked in 2 teams of 2 collectors each. One team worked from 18.00 until $24.00 \mathrm{~h}$ with one individual collecting the mosquitoes which landed on him inside a house and the other individual collecting outside. This team was relieved by the second team at $24.00 \mathrm{~h}$, which worked until $06.00 \mathrm{~h}$. Searches for blood-engorged anophelines resting on the walls and furniture of all houses in the village began at 06.00 and finished by $07.00 \mathrm{~h}$.

Mosquito collections were transported to the laboratory in pint containers inside a styrofoam insulated container. Mosquitoes from resting collections were held at ambient temperature for 5-6 days before dissection, while mosquitoes from biting collections were dissected immediately.

Dissections were performed by a team of 4 individuals. The first individual identified the mosquito species by morphological criteria (Belkin, 1962 ) and then removed the stomach into a $0.2 \%$ solution of mercurochrome. Stomachs were examined under $100 \times$ and $400 \times$ magnification for oocysts by the second team member. Salivary glands were removed by the third member and placed into mosquito grinding buffer for $P$. falciparum and $P$. vivax sporozoite antigen analysis by enzymelinked immunosorbent assay (ELISA) (Wirtz et al. 1987, 1985). The remaining mosquito material from landing catches was placed into a $1.5 \mathrm{ml}$ vol. Eppendorf microcentrifuge tube filled with $70 \%$ ethanol, and later stained in Mayer's acid haemalum and dissected for the presence of $W$. bancroft $i$ larvae by the fourth member of the team (Nelson, 1959). Mosquitoes from resting catches were dissected immediately by the fourth member of the team and examined at $400 \times$ magnification for filarial larvae.

Dissection results for anophelines collected in biting and resting collections were analysed separately because mosquitoes collected resting had had one more opportunity to become infected than mosquitoes from biting collections.

The mosquito infection probability was determined as described previously (Graves et al. 1988). Briefly, laboratory reared $A n$. farauti were allowed to engorge on volunteers between 22.00 and $24.00 \mathrm{~h}$. Immediately prior to feeding mosquitoes, blood films were made for malaria parasite examination and $2 \mathrm{ml}$ vol. blood samples were collected and filtered for microfilaria density determinations. Engorged mosquitoes were held in the insectary for 6 days and then dissected for oocysts and Stage 2 larvae as described above.

\section{RESULTS}

The prevalences of malaria and $W$. bancrofti infections in $A n$. punctulatus in Buksak village are presented in Table 1. Malaria infection rates for all species are given together. Significantly greater infection rates for both malaria and filariae were found in anophelines captured in resting compared to biting catches. The expected number of multiple infections was calculated from the observed frequencies of malaria and filaria infections for each 
Table 1. Prevalence of naturally acquired infections of oocysts and sporozoites of Plasmodium falciparum (PF) and $P$. vivax (PV) and Wuchereria bancrofti Stage 1, 2 and 3 larvae (L1, L2, L3) in Anopheles punctulatus

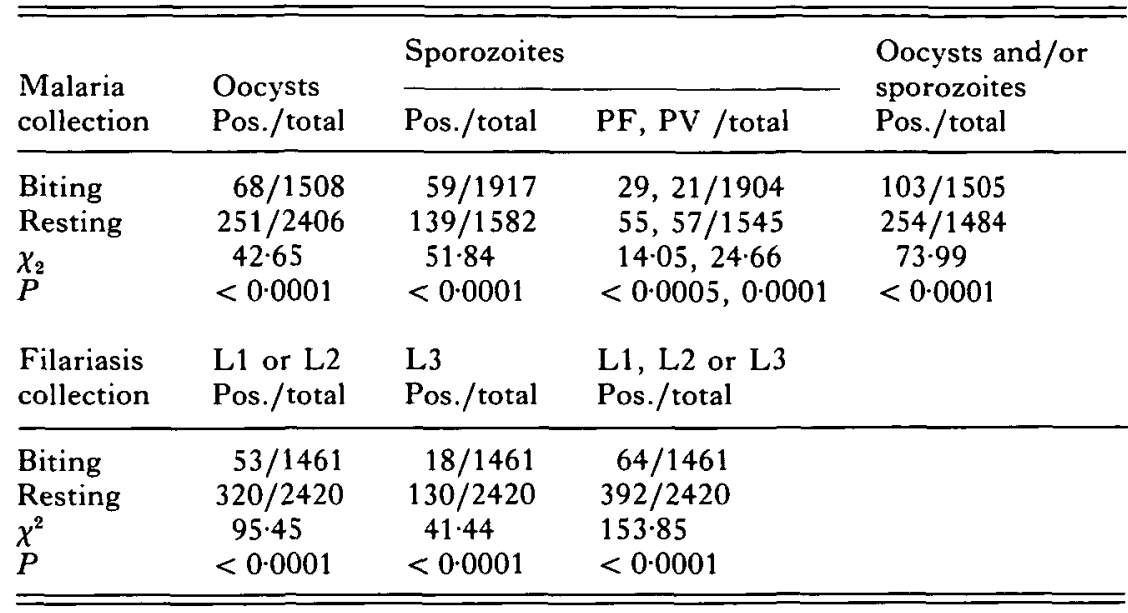

Table 2. Analysis of the numbers of multiple infections involving human malaria oocysts, sporozoites of Plasmodium falciparum (PF) and $P$. vivax (PV), and Stage 1 (L1), Stage 2 (L2) and Stage 3 (L3) larvae of Wuchereria bancrofti

\begin{tabular}{|c|c|c|c|c|c|c|c|}
\hline & \multicolumn{4}{|c|}{ Biting collection } & \multicolumn{3}{|c|}{ Resting collection } \\
\hline & $\mathrm{Ob}$ & Exp. & (Total) & $P^{*}$ & Obs. & Exp. & (Total) $P^{*}$ \\
\hline Oocysts and L3 & 3 & 0.8 & $(1360)$ & 0.223 & 25 & $13 \cdot 4$ & (2400) 0.086 \\
\hline Sporozoites and L1, L2 & 5 & $1 \cdot 6$ & (1454) & $0 \cdot 137$ & 28 & $17 \cdot 4$ & (1498) 0.151 \\
\hline Oocysts and $\mathrm{L} 1, \mathrm{~L} 2$ & 7 & $2 \cdot 2$ & (1360) & 0.079 & 49 & $33 \cdot 1$ & (2400) 0.097 \\
\hline Sporozoites and L3 & 0 & 0.6 & (1454) & $0 \cdot 160$ & 20 & $7 \cdot 1$ & (1498) 0.022 \\
\hline Sporozoites PF and PV & 4 & $0 \cdot 3$ & (1904) & 0.090 & 10 & $2 \cdot 0$ & (1545) 0.016 \\
\hline $\begin{array}{l}\text { Malaria and Filariasis } \\
\text { Overall }\end{array}$ & 9 & $2 \cdot 9$ & $(1357)$ & $0 \cdot 051$ & 71 & $41 \cdot 0$ & (1480) $0 \cdot 005$ \\
\hline
\end{tabular}

* Analysis by Fisher's Exact Test.

collection type and are presented in Table 2 together with numbers of multiple infections observed by dissection.

A larger number of multiple infections was observed than predicted for almost all categories of infection and type of collections. This difference reached statistical significance in resting catches for the numbers of mosquitoes infected with malaria sporozoites and $W$. bancrofti Stage 3 larvae $(P<0.05$, Fisher's Exact Test $)$ and for those mosquitoes infected with $P$. falciparum and $P$. vivax sporozoites $(P<0.02$, Fisher's Exact Test). In addition, the number of mixed malaria and filariae vector infections for all combinations of stages was significantly greater than predicted $(P<0.01$, Fisher's Exact Test).

In comparing parasite burdens in singly and multiply infected $A n$. punctulatus, a significantly greater geometric mean number of Stage 3 larvae was found in mosquitoes collected whilst resting indoors that were also infected with malaria than in mosquitoes without a simultaneous malaria infection (Unpaired $t$-test: $t=2.51, \quad$ D.F. $=104, P<0.02$ ) (Table 3). No significant differences in oocyst densities were found between filariae-positive and negative anophelines.

Comparisons between parasite burdens in mosquitoes from resting and landing collections revealed a statistically greater geometric mean number of Stage 3 and total $W$. bancrofti larvae in $A n$. punctulatus from resting compared to landing catches (Unpaired $t$-test: $t=2.25,4.84 ; P<0.05$ and $P<0.001$, respectively).

Laboratory reared An. farauti engorged on 43 residents of Buksak village; 16 and 22 individuals were malaria and microfilaria positive, respectively, with 8 persons harboring both parasites. The numbers of individuals dually infected did not differ significantly from expected $\left(x^{2}=0.05, P>0.80\right)$. Of the 16 malaria-positive individuals 5 were infectious to mosquitoes and 11 of 22 microfilaraemic individuals were infectious. None were infectious for 
Table 3. Geometric mean infection (GM) loads of oocysts and Wuchereria bancrofti larvae in Anopheles punctulatus in single and multiple infections

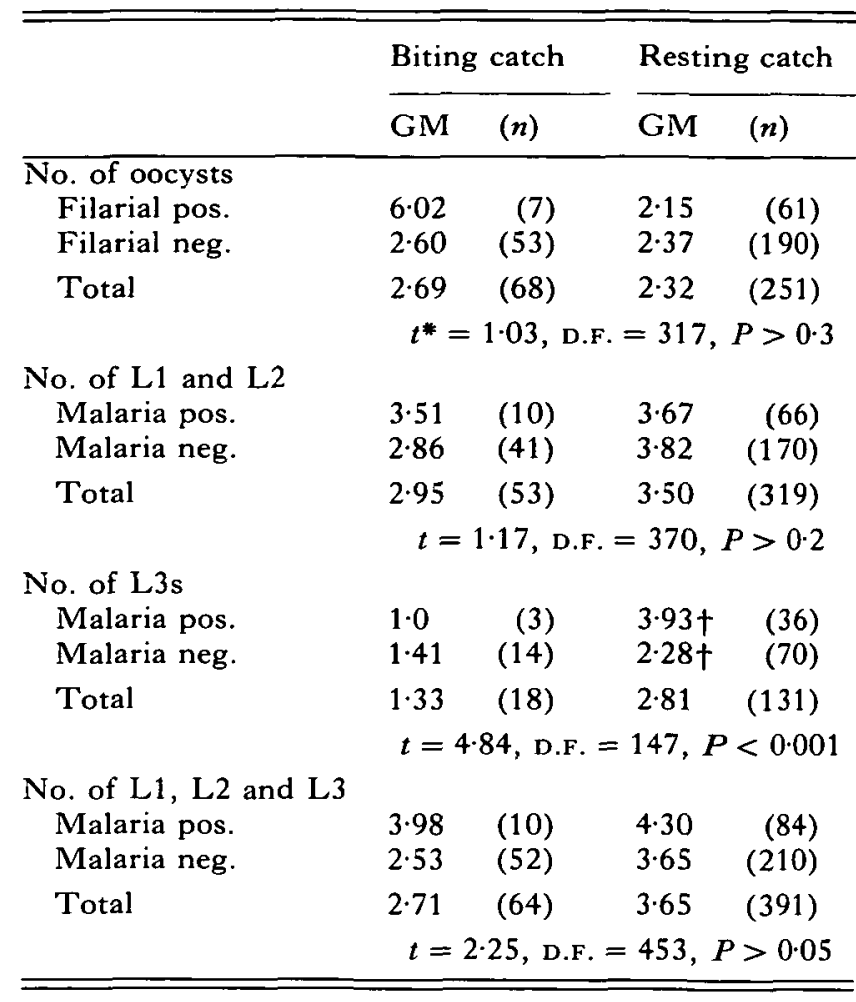

* Comparison of biting and resting catches by unpaired $t$ test.

† Unpaired $t$-test: $t=2 \cdot 51$, D.F. $=104, P<0.02$.

both malaria and filaria, which was not significantly different from expected $(P>0 \cdot 40$, Fisher's Exact Test).

\section{DISCUSSION}

The greater malaria and filariae infection rates found in resting mosquitoes than biting mosquitoes result from three factors: (1) mosquitoes from resting collections were held in the insectary until all parasites have developed to a size easily detectable by light microscopy, (2) mosquitoes resting indoors had one more opportunity to feed on an infectious individual than mosquitoes collected in the process of biting a malaria and filariasis aparasitaemic collector, and (3) infected mosquitoes collected in resting catches had a greater opportunity to survive as they were held in an unstressful environment for 5-6 days before dissection.

Probability analysis of multiple malaria and filaria infections in mosquitoes from resting catches indicates that multiple parasite species infections are more frequent than one would predict from the prevalence of single-species infections. Supporting evidence for this conclusion is the significantly greater numbers of multiple infections in resting mosquitoes than expected from the product of the overall infection rates of malaria and filariae. Although not statistically significant in the biting catches, a larger number of multiple infections were observed for almost all combinations of parasite stages than predicted. The lack of significance is probably a function of relatively small sample sizes $(n=1357-1904)$ coupled with relatively low mosquito survivorship and infection probabilities (Burkot et al. manuscript submitted; Graves et al. 1989).

There are several possible explanations for the greater observed than expected multiple infection rates in mosquitoes including: (1) greater multiple infection rates in humans, (2) preferential selection of parasitized humans by anophelines (Appendix 1), (3) preferential capture of multiply infected vectors by collectors, (4) a vector population composed of individuals with different numbers of opportunities to have fed (e.g. a population non-homogeneous for age or host preference and/or one exhibiting interrupted feeding habits, Appendix 2) and (5) enhanced susceptibility of these vectors to malaria infections following physical disruption of the mosquito stomach by microfilariae.

The first three of these possibilities are improbable or unsubstantiated based on the data presently available. Experiments on the infectivity of the human population to anophelines revealed no significant difference in the number of dual malaria - microfilaria infected humans than expected from the overall infection rates. However, this observation was based on the relatively insensitive detection of blood-stage malaria parasites by light microscopy. As an anopheline ingests far more blood than a microscopist examines, mixed infections which suppress the parasitaemia of one of the parasites may lead to under-estimation of the true incidence of that parasite and therefore an underestimate of the incidence of multiple infections in humans.

There is no evidence for the second possibility, preferential selection of infected individuals by anophelines. Although theoretical analysis (Appendix 1) predicts that an excess of multiply infected vectors will result from multiply infectious humans being more attractive to mosquitoes than the product of the attractiveness of singly infected humans, field experiments have demonstrated that single and multiple infected humans are no more likely to be fed upon than uninfected individuals in this hyperendemic area (Burkot et al. 1989).

Preferential capture of vectors with multiple parasite infections might result in an excess of multiply infected vectors. The laboratory based findings that flight behaviour is affected by filarial infections (Townson, 1970; Hockmeyer et al. 1975; Berry et al. 1987; Rowland \& Boersma, 1988) might lead to preferential capture of infected mosquitoes. 
The theoretical analysis is similar to that for preferential selection of parasitized hosts (Appendix 1 ), but no field data are yet available to validate this hypothesis.

The fourth possibility, a vector population composed of individuals with different numbers of opportunities to become infected, cannot be dismissed and is, in fact, quite likely to be responsible for the excess of multiply infected mosquitoes observed (Appendix 2). A vector population composed of individuals differing in the number of feeding cycles is the norm. In addition, interrupted feeding on humans is known to occur in the members of the Anopheles punctulatus complex at a rate between 0.13 and 0.41 (Burkot et al. 1988b). Such interrupted feeding will contribute to different numbers of opportunities for mosquitoes to become infected.

The fifth possible explanation for enhanced multiple vector infections, physical disruption of the gut by microfilariae (Perrone $\&$ Spielman, 1986) thereby removing it as a significant barrier to malaria infection may also contribute to the greater multiple infected rate. Evidence for this was found in a microfilariae-virus model (Turrell et al. 1984) and support for this hypothesis is seen in the significantly greater mean number of Stage 3 larvae found in mosquitoes concurrently infected with malaria. However, any advantages to pathogen transmission by enhanced susceptibility to multiple infections in mosquitoes appear to be offset by an increased mortality rate due to heavy and/or multiple infections (Bryan, 1986).

The authors wish to thank Dr Joan Bryan for reviewing the manuscript, Dr Karen Forsyth for filtering blood samples for microfilaria, and especially the members of the Papua New Guinea Institute of Medical Research for their expert assistance, particularly Moses Lagog and the members of the Entomology Department. T. R. Burkot and A. Barnes were supported, in part, by the Tropical Health Program and P. M. Graves was supported by the Wellcome Trust. Reagents used in this study were produced under United Nations Development Programme/World Bank/WHO Special Programme for Research and Training Grant 880068.

\section{APPENDICES}

Some of the hypotheses suggested as possible explanations of the observed excess of mixed infections have been explored by comparing the frequency of mixed infections generated by models incorporating the hypotheses (frequency hereafter called 'observed') to the product of the frequencies of individual infections (pure or mixed) generated by the same models (product hereafter called 'expected').

\section{APPENDIX 1}

\section{Non-random host selection and the frequency of multiple infections in the vector}

Let 2 parasite infections be randomly distributed in the human population with frequencies $p_{1}$ and $p_{2}$, and let $1-p_{1}=q_{1}$ and $1-p_{2}=q_{2}$. The frequency of double negatives is $q_{1} q_{2}$, that of single positives of the first and second parasite is $p_{1} q_{2}$ and $p_{2} q_{1}$, that of double positives is $p_{1} p_{2}$. Let the relative attractiveness (for vectors) of the 4 classes be $1,\left(1+c_{1}\right),\left(1+c_{2}\right)$, and $\left(1+c_{12}\right)$, where $c_{12}>c_{1}, c_{2}>0$ (i.e. double positives are more attractive than single positives, which are more attractive than double negatives). The relative frequency, among vectors taking a human blood meal of double negatives is $q_{1} q_{2}$, that of single positives of the first and second kind is $\left(1+c_{1}\right) p_{1} q_{2}$ and $\left(1+c_{2}\right) p_{2} q_{1}$, that of double positives is $\left(1+c_{12}\right) p_{1} p_{2}$. These 4 frequencies are normalized by dividing each of them by the sum of all 4 . The comparison between 'observed' and 'expected' can be formulated as follows:

$$
\begin{aligned}
& \text { ? } \\
& \text { Observed }><\text { Expected } \\
& \left\{\left(1+c_{12}\right) p_{1} p_{2} /\left[q_{1} q_{2}+\left(1+c_{1}\right) p_{1} q_{2}\right. \text { ? }\right. \\
& \left.\left.+\left(1+c_{2}\right) p_{2} q_{1}+\left(1+c_{12}\right) p_{1} p_{2}\right]\right\}><\left\{\left[\left(1+c_{1}\right) p_{1} q_{2}+\left(1+c_{12}\right) p_{1} p_{2}\right]\right. \\
& \times\left[\left(1+c_{2}\right) p_{2} q_{1}+\left(1+c_{12}\right) p_{1} p_{2}\right] \\
& \div\left[q_{1} q_{2}+\left(1+c_{1}\right) p_{1} q_{2}\right. \\
& \left.\left.+\left(1+c_{2}\right) p_{2} q_{1}+\left(1+c_{12}\right) p_{1} p_{2}\right]^{2}\right\} \\
& \text { which simplifies to } \quad\left(1+c_{12}\right)><\left(1+c_{1}\right)\left(1+c_{2}\right) \\
& \text { i.e. Observed }>\text { Expected if } \\
& \text { Observed }<\text { Expected if } \\
& \left(1+c_{12}\right)>\quad\left(1+c_{1}\right)\left(1+c_{2}\right) \\
& \left(1+c_{12}\right)<\quad\left(1+c_{1}\right)\left(1+c_{2}\right) .
\end{aligned}
$$

Preferential feeding on infected persons produces an excess of double infections in the vector only if the attractiveness of the host for vectors of double positives exceeds the product of the attractiveness of the two kinds of single positives. Below that threshold, preferential selection on infected persons will produce a deficit of double infections. 


\section{APPENDIX 2}

The consequences of different numbers of blood meals in the vector population

Let $p_{1}, p_{2}, q_{1}, q_{2}$ and the frequencies of single and double infections in the human population be defined as in Appendix 1, but let vectors feed at random.

Consider first a vector population that has taken $n$ meals. The frequency of double negatives is $q_{1}^{n} q_{2}^{n}$, that of single positives of the first and second parasite are $\left(1-q_{1}^{n}\right) q_{2}^{n}$ and $\left(1-q_{2}^{n}\right) q_{1}^{n}$, that of double positives can be obtained by subtraction. The total frequency of parasite 1 (pure or mixed) is $\left(1-q_{1}^{n}\right)$, that of parasite 2 is $\left(1-q_{2}^{n}\right)$. The comparison between 'observed' and 'expected' can be formulated as follows:

$$
\begin{gathered}
\text { Observed }> \\
><\text { Expected } \\
1-q_{1}^{n} q_{2}^{n}-\left(1-q_{1}^{n}\right) q_{2}^{n}-\left(1-q_{2}^{n}\right) q_{1}^{n}><\left(1-q_{1}^{n}\right)\left(1-q_{2}^{n}\right) .
\end{gathered}
$$

It is easy to show that the observed and expected are equal, i.e. among vectors that took the same number of bloodmeals, there is neither excess nor deficit of mixed infections (and the formula for the 'expected', which is simpler, can be used to describe the 'observed' as follows).

Now consider a vector population made of individuals of mixed numbers of feeds. To simplify matters, assume that the population is divided equally among individuals having taken ' $n$ ' and ' $n+x$ ' feeds where $x \geqslant 1$. In the first half of the population, frequencies are as above. Substituting $(n+x)$ for $n$ yields the corresponding frequencies in the second half of the population. The comparison between observed and expected, in the population as a whole, can be formulated as follows:

$$
\begin{aligned}
& 0 \cdot 5\left[\left(1-q_{1}^{n}\right)\left(1-q_{2}^{n}\right)+\left(1-q_{1}^{n+x}\right)\left(1-q_{2}^{n+x}\right)\right] \stackrel{?}{>}<0 \cdot 5\left[\left(1-q_{1}^{n}\right)+\left(1-q_{1}^{n+x}\right)\right] 0 \cdot 5\left[\left(1-q_{2}^{n}\right)+\left(1-q_{2}^{n+x}\right)\right] \\
& \text { which simplifies to } \quad q_{1}^{n} q_{2}^{n}+q_{1}^{n+x} q_{2}^{n+x}><q_{1}^{n} q_{2}^{n+x}+q_{1}^{n+x} q_{2}^{n} \\
& \text { or } 1+q_{1}^{x} q_{2}^{x}><q_{2}^{x}+q_{1}^{x} \\
& \text { or } \quad\left(1-q_{1}^{x}\right)\left(1-q_{2}^{x}\right)><0 \text {. }
\end{aligned}
$$

Given that $q_{1}, q_{2}<1$ and $x \geqslant 1+$, it is obvious that the left-hand side is larger than zero, i.e. mixing vectors that have taken different numbers of feeds generates an 'excess' of mixed infections.

\section{REFERENCES}

BELkin, J. N. (1962). The Mosquitoes of the South Pacific (Diptera, Culicidae), vol. 1. pp. 123-47. Berkeley: University of California Press.

BERRY, W. J., ROWLEY, W. A. \& CHRISTENSEN, B. M. (1987). Influence of developing Dirofilaria immitis on the spontaneous flight activity of Aedes aegypti (Diptera: Culicidae). Journal of Medical Entomology 24, 699-701.

BRYAN, J. H. (1986). Vectors of Wuchereria bancrofti in the Sepik Provinces of Papua New Guinea.

Transactions of the Royal Society of Tropical Medicine and Hygiene 80, 123-31.

BURKot, T. R., GRAVES, P. M., CATTANI, J. A., WIRTZ, R. A. \& GIBSON, F. D. (1987). The efficiency of sporozoite transmission of the human malarias, Plasmodium falciparum and $P$. vivax. Bulletin of the World Health Organization 65, 375-80.

BURKOT, T. R., GRAVES, P. M., PARU, R. \& LAGOG, M. $(1988 b)$. Mixed blood feeding by the malaria vectors in the Anopheles punctulatus complex (Diptera: Culicidae). Fournal of Medical Entomology 25, 205-13. BURKot, T. R., GRAVES, P. M., PARU, R., WIRTZ, R. A. \& HEYWOOD, P. (1988 a). Human malaria transmission studies in the Anopheles punctulatus complex in Papua New Guinea: sporozoite rates, inoculation rates and sporozoite densities. American fournal of Tropical Medicine and Hygiene 39, 135-44.

BURKot, T. R., NARARa, A., PARU, R., GRAVES, P. M. \&
GARNER, P. (1989). Human host selection by anophelines: no evidence for preferential selection of malaria or microfilariae infected individuals in a hyperendemic area. Parasitology 97, 337-42.

COLEMAN, R. E., EDMAN, J. D. \& SEMPREVIVO, L. H. (1988). Interactions between malaria (Plasmodium yoelii) and leishmaniasis (Leishmania mexicana amazonensis): effect of concomitant infection on host activity, host body temperature, and vector engorgement success. Fournal of Medical Entomology 25, 467-71.

Cox, F. E. G. (1978). Concomitant infections. In Rodent Malaria (ed. Killick-Kendrick, R. \& Peters, W.), pp. 309-43. London: Academic Press.

HOCKMEYER, W. T., SCHIEFFER, B. A., REDINGTON, B. C. \& ELDRIDGE, B. F. (1975). Brugia pahangi: effects upon the flight capability of Aedes aegypti. Experimental Parasitology 38, 1-5.

GRaVes, P. M., BURKot, T. R., CARTER, R., CATTANi, J. A., LAGOG, M., PARKER, J., BRABIN, B. J., GIBSON, F. D., BRADLEY, D. J. \& ALPERS, M. P. (1988). Measurement of malarial infectivity of human populations to mosquitoes in the Madang area, Papua New Guinea. Parasitology 96, 251-63.

GRAVES, P. M., BURKoT, T. R., SAUl, A., haYes, R. \& CARTER, R. (1989). Estimation of anopheline survival rate, vectorial capacity, and mosquito infection probability from malaria vector infection rates in villages near Madang, Papua New Guinea. Yournal of Applied Ecology 26 (in the Press).

Javadian, E. \& MaCDONaLd, w. w. (1974). The effect of 
infection with Brugia pahangi and Dirofilaria repens on the egg production of Aedes aegypti. Annals of Tropical Medicine and Parasitology 68, 477-81.

KLEIN, T. A., HARRISON, B. A., GROVE, J. S., DIXON, S. v. \& ANDRE, R. G. (1986). Correlation of survival rates of Anopheles dirus A (Diptera: Culicidae) with different infection densities of Plasmodium cynomolgi. Bulletin of the World Health Organization 64, 901-7.

MilloTt, S. M. \& coX, F. E. G. (1985). Interactions between Trypanosoma brucei and Babesia spp. and Plasmodium spp. in mice. Parasitology 90, 241-54.

MOLineauX, L., STOREY, J., COHEN, J. E. \& THOMAS, A. (1980). A longitudinal study of human malaria in the west African savanna in the absence of control measures: relationships between different Plasmodium species, in particular $P$. falciparum and $P$. malariae. American Fournal of Tropical Medicine and Hygiene 29, 725-37.

NeLSON, G. S. (1959). The identification of infective filarial larvae in mosquitoes: with a note on the species found in 'wild' mosquitoes on the Kenya Coast. Fournal of Helminthology 33, 233-56.

PERRONE, J. B. \& SPIELMAN, A. (1986). Microfilarial perforation of the midgut of a mosquito. Journal of Parasitology 72, 723-7.

PONNudurai, T., BILlingsley, P. F. \& RUDIN, w. (1988). Differential infectivity of Plasmodium for mosquitoes. Parasitology Today 4, 319-21.

RICHIE, T. L. (1988). Interactions between malaria parasites infecting the same vertebrate host. Parasitology 96, 607-39.

ROWLAND, M. \& BOERSMA, E. (1988). Changes in the spontaneous flight activity of the mosquito Anopheles stephensi by parasitization with the rodent malaria Plasmodium yoelii. Parasitology 97, 221-7.

SCHMIDT, L. H. \& ESSINGER, J. H. (1981). Courses of infection with Plasmodium falciparum in owl monkeys displaying a microfilaremia. American fournal of Tropical Medicine and Hygiene 30, 5-11.

Townson, H. (1970). The effect of infection with Brugia pahangi on the flight of Aedes aegypti. Annals of Tropical Medicine and Parasitology 64, 411-20.

TOWNSON, H. (1971). Mortality of various genotypes of the mosquito Aedes aegypti following the uptake of microfilariae of Brugia pahangi. Annals of Tropical Medicine and Parasitology 65, 93-106.

TURRELL, M. J., ROSSINGNOL. P. A., SPIELMAN, A., ROSSI, C. A. \& BAILEY, C. L. (1984). Enhanced arboviral transmission by mosquitoes that concurrently ingested microfilariae. Science 225, 1039-41.

Williams, R. B. (1985). Plasmodium gallinaceum and subsequent Eimeria acervulina infections in chickens are not synergistic. Parasitology 90, 237-40.

WIRTZ, R. A., BURKOT, T. R., ANDRE, R. G., ROSENBERG, R., COLLINS, W. E. \& ROBERTS, D. R. (1985). Identification of Plasmodium vivax sporozoites in mosquitoes using an enzyme-linked immunosorbent assay. American Fournal of Tropical Medicine and Hygiene 34, 1048-54. WIRTZ, R. A., ZAVALA, F., CHAROENVIT, Y., CAMPBELl, G. H., BURKOT, T. R., SCHNEIDER, I., ESSER, K. M., BEAUDOIN, R. L. \& ANDRE, R. G. (1987). Comparative testing of Plasmodium falciparum sporozoite monoclonal antibodies for ELISA development. Bulletin of the World Health Organization 65, 39-45. 